\title{
GENETIC POLYMORPHISM WITHIN THE LEISHMANIA DONOVANI COMPLEX: CORRELATION WITH GEOGRAPHIC ORIGIN
}

\author{
EVA ZEMANOVÁ, MILAN JIRKŮ, ISABEL L. MAURICIO, MICHAEL A. MILES, AND JULIUS LUKEŠ \\ Institute of Parasitology, Czech Academy of Sciences, and Faculty of Biology, University of South Bohemia, České Budějovice, \\ Czech Republic; Pathogen Molecular Biology Unit, Department of Infectious and Tropical Diseases, London School of Hygiene and \\ Tropical Medicine, London, United Kingdom
}

\begin{abstract}
Random amplified polymorphic DNA (RAPD) was used to detect intraspecific diversity for the Leishmania donovani complex. Fifty-two decameric to 21-meric primers of arbitrary sequence were applied to 15 strains that belong to nine zymodemes. Strains belonging to the species L. major and L. tropica were used as outgroups. A total of 902 amplicons generated by RAPD were scored. Most primers produced species-specific profiles, only $0.6 \%$ amplicons were shared by all species, while $4.3 \%$ amplicons were common for all 15 strains of the L. donovani complex. Wellsupported trees have been constructed, which show a rather strong correlation between the genetic polymorphism of studied strains and their geographic origin. In all obtained trees, L. infantum was paraphyletic. The RAPD profiles suggest that MON-30 belongs to L. donovani. Moreover, the genetic distance between the L. archibaldi strain and other leishmanias does not warrant existence of a separate species.
\end{abstract}

\section{INTRODUCTION}

The Leishmania donovani complex, the etiologic agent of visceral leishmaniasis, is reported to include three species $(L$. donovani, L. infantum, and $L$. archibaldi), the concept of which is based on multilocus enzyme electrophoresis. ${ }^{1,2}$ However, this method has several shortcomings, ${ }^{3}$ and is being questioned by various DNA-based methods: sequencing of ribosomal intergenic regions, ${ }^{4}$ mini-exons, ${ }^{5}$ and gp63 genes, ${ }^{6}$ microsatellite analysis, ${ }^{7}$ kinetoplast DNA minicircles, ${ }^{8}$ and polymerase chain reaction-restriction fragment length polymorphism of gp63. ${ }^{9,10}$ Data from these analyses have been used to build phylogenetic trees, which in general did not correlate with taxonomy based on multilocus enzyme electrophoresis. However, DNA-based phylogenies were all derived from a small number of characteristics and a high level of genetic homogeneity within the complex makes it rather difficult to reconstruct (robust) phylogenetic relationships. For comparison of closely related Leishmania strains, the limited reproducibility of random amplified polymorphic DNA (RAPD) seems to be outweighed by the amount of information obtained. The RAPDs have provided the most polymorphic characteristics, but have been applied with only a small number of primers. Nevertheless, RAPD has enabled the detection of intraspecific diversity for L. donovani and L. infantum. .,11-14 $^{-14}$

Here we present a RAPD analysis of the L. donovani complex using 52 primers that provided numerous distinct amplicon profiles. Well-supported RAPD based trees of this complex were obtained for the first time and revealed a significant correlation between genetic polymorphism and geographic origin in L. infantum/donovani.

\section{MATERIALS AND METHODS}

Total DNA was obtained from 15 L. donovani complex strains $(10 \mathrm{~L}$. infantum and $5 \mathrm{~L}$. donovani; full strain information at http://www.paru.cas.cz/structure/LMP/leish.htm), one L. major strain, and one L. tropica strain (Table 1) isolated from autochthonous patients in different geographic regions, using a DNA isolation kit (Qiagen, Valencia, CA) according to the manufacturer's instructions. Amplicons were obtained with 62 random decameric to 21-meric primers
(Table 2). Each $25-\mu \mathrm{L}$ RAPD reaction contained $5 \mathrm{ng}$ of genomic DNA, $3 \mathrm{mM} \mathrm{MgCl}_{2}, 25 \mathrm{pmol}$ of primer, and 0.5 units of $\mathrm{Taq}$ polymerase in the appropriate buffer. The amplification cycle was $94^{\circ} \mathrm{C}$ for four minutes; then 45 cycles at $94^{\circ} \mathrm{C}$ for one minute, $35^{\circ} \mathrm{C}$ for two minutes, and $72^{\circ} \mathrm{C}$ for three minutes; and at $72^{\circ} \mathrm{C}$ for 10 minutes. The RAPD products were separated by electrophoresis on ethidium bromide-stained $1.5 \%$ agarose gels in $0.5 \times$ Tris-acetate-EDTA buffer (20 mM Tris-acetate, $0.5 \mathrm{mM}$ EDTA) at $80 \mathrm{~V}$ for four hours. The size of the scored amplicons ranged from 100 basepairs to 4 kilobases.

Fifty-two primers had a sufficient number of scorable amplicons and good reproducibility, tested by repeating most reactions. Although profiles were reproducible, the intensity of individual bands was subject to significant differences, which may be due to small differences in DNA and primer concentrations. High-quality DNA was always used because degradation of DNA altered amplification profiles.

The RAPD profiles were manually scored as presence or absence data. Phylogenetic trees were constructed using minimum evolution as an optimality criterion and NeiLi or UpHolt distance matrices (as implemented in PAUP 4b10, Swofford, 1998; Sinauer Associates, Sunderland, MA).

\section{RESULTS}

Forty-three primers were used with all strains shown in Table 1. Figure 1 shows a representative RAPD gel (other gels are available at http://www.paru.cas.cz/structure/LMP/ Figures.htm) and each generated between 1 and 34 amplicons scored by size, in a total of 770 . Nine additional primers were used with a smaller set of strains and produced 132 scored amplicons. Thus, a total of 902 amplicons has been evaluated. Scores obtained with individual primers are available at http://www.paru.cas.cz/structure/LMP/RAPD_primers.htm. Most primers produced profiles specific for $L$. major, $L$. tropica, or L. aethiopica, and the L. infantum/L. donovani complex. The length of the primers did not correlate with the number of genetic markers. Only $0.6 \%$ of the amplicons were shared by all four species, while $4.3 \%$ were common for the 15 strains of the L. donovani complex. When this complex was subdivided into $L$. infantum and $L$. donovani (based on multilocus enzyme electrophoresis), ${ }^{1,2} 16.6 \%$ and $6.9 \%$ were species specific, respectively. 
TABLE 1

Characterization of analyzed Leishmania spp. strains*

\begin{tabular}{|c|c|c|c|c|c|}
\hline Name & Code & Strain & Zymodeme & Country & $\begin{array}{l}\text { Type of } \\
\text { infection }\end{array}$ \\
\hline L. infantum & LG1 & MHOM/FR/1978/LEM75 & MON1 & France & VL \\
\hline L. infantum & LG2 & MHOM/FR/1995/LPN114 & MON1 & France & VL \\
\hline L. infantum & LG3 & MHOM/1993/PM1 & MON1 & Spain & VL \\
\hline L. infantum & LG4 & MHOM/FR/1997/LSL29 & MON1 & France & CL \\
\hline L. infantum & LG5 & MHOM/ES/1986/BCN16 & MON1 & Spain & $\mathrm{CL}$ \\
\hline L. infantum & LG6 & MHOM/PT/2000/IMT260 & MON1 & Portugal & CL \\
\hline L. infantum & LG7 & MHOM/FR/1996/LEM3249 & MON29 & France & VL \\
\hline L. infantum & LG8 & MHOM/ES/1991/LEM2298 & MON183 & Spain & VL \\
\hline L. donovani & LG9 & MHOM/IN/0000/DEVI & MON2 & India & VL \\
\hline L. donovani & LG10 & MHOM/IN/1996/THAK35 & MON2 & India & VL \\
\hline L. archibaldi & LG11 & MHOM/ET/1972/GEBRE 1 & MON82 & Ethiopia & VL \\
\hline L. donovani & LG12 & MHOM/SD/1982/GILANI & MON30 & Sudan & VL \\
\hline L. donovani & LG13 & MHOM/ET/0000/HUSSEN & LON42 & Ethiopia & VL \\
\hline L. infantum & LG14 & MHOM/FR/1980/LEM189 & MON11 & France & VL \\
\hline L. infantum & LG15 & MHOM/MT/1985/BUCK & MON78 & Malta & VL \\
\hline L. major & M & LV561 & $?$ & Israel & CL \\
\hline L. tropica & $\mathrm{T}$ & VEDHA & $?$ & Turkey & CL \\
\hline
\end{tabular}

$* \mathrm{VL}=$ visceral leishmaniasis; $\mathrm{CL}=$ cutaneous leishmaniasis.

The power to discriminate between the L. donovani complex and the outgroups (L. tropica or L. aethiopica and $L$. major) was primarily observed for primers A12, B13, ILO875, and TA-150. Seven primers (A5, B5, B6, B7, B8, 198, and TA-150) distinguished $L$. infantum from $L$. donovani strains. Selected amplicons of varying specificity, obtained with the above-mentioned primers, are being cloned and sequenced with the aim of obtaining species-specific regions. The profiles most polymorphic for the strains LG1 to LG8 ( $L$. infantum sensu stricto) were generated by primers B1, B3, B9, B10, B20, and 193. Moreover, primers A15, B12, and TA-150 distinguished between Indian L. donovani and Mediterranean $L$. infantum. Importantly, none of the primers used distinguished between $L$. infantum, L. donovani, and L. archibaldi. Moreover, primer H1 produced a $L$. donovani-specific band of approximately 600 basepairs.

Phylogenetic trees were constructed using the following doublets of strains (Table 1) as outgroups: 1) L. major and $L$. tropica; 2) strains DEVI and THAK 35 (L. donovani from India); and 3) strains LEM 189 and BUCK (L. infantum). Overall, the analysis showed well-resolved trees with topology slightly influenced by the choice of outgroup. When the tree was rooted with $L$. major and $L$. tropica, $L$. infantum strains LEM 189 and BUCK formed a sister group to the remaining strains (Figure 2) with the longest interior branches. The three African strains were a strongly supported group ( $86 \%$ bootstrap), the relationship of which with the clade consisting of Indian L. donovani was not fully resolved (Figure 2). The crown group, composed solely of strains isolated from humans originating from the Iberian peninsula, was supported by $98 \%$ bootstrap with this outgroup.

Exclusion of L. major and L. tropica from the dataset reduced the number of non-shared characteristics and thus increased bootstrap support. Almost no differences were observed between the UpHolt and NeiLi matrices. Upon rooting with LEM 189 and BUCK, the first branch was composed of African strains, followed by one with both Indian L. donovani strains (Figure 3A). Leishmania donovani strains isolated from India and Sudan were clearly paraphyletic. The crown group (bootstrap support 98\%) contained Mediterranean strains (Figure 3A).
Trees rooted with DEVI and THAK 35 (Indian L. donovani) were split into two strongly supported branches, with internal topology being apparently insensitive to the outgroup selected (Figure 3B). Leishmania donovani strains were not monophyletic in any of the analyzed trees, nor were the $L$. infantum MON-1 strains. Interestingly, all three MON-1 strains isolated from cutaneous forms of the disease formed a clade, albeit also containing one strain from a visceral case (Figures 2 and 3).

In a separate RAPD analysis with a higher number of strains but with less primers (C2, C4, C5, C6, D8, D10, H1, $\mathrm{H} 4$, and L2) using conditions previously described, ${ }^{6}$ all primers distinguished the L. donovani complex from outgroup strains (L. major and L. aethiopica), while primers $\mathrm{C} 4$ and $\mathrm{H} 1$ produced $L$. infantum-specific profiles. In the resultant trees, two groups, L. infantum strains and $L$. donovani strains originating from India, were robust and a group with strains of Kenyan origin was detected but not strongly supported. Strains from Ethiopia/Saudi Arabia or Sudan did not form monophyletic assemblies.

\section{DISCUSSION}

The RAPD analysis of strains of the L. donovani complex presented here detected a correlation with geographic origin, regardless of whether a high number of studied strains or primers was used in a given dataset. With a single exception, L. infantum strains originating from France, Spain, and Portugal were strongly monophyletic. Moreover, the African and Indian strains constituted two monophyletic groups.

Strain Gilani (MON-30) did not cluster with L. infantum, as it would be expected from the report of Rioux and others, ${ }^{1}$ but with Gebre 1 (MON-82). This suggests that strains MON30 are not $L$. infantum and casts doubts on the existence of the species $L$. archibaldi. Along with gp63 restriction fragment length polymorphism data, ${ }^{9}$ recently obtained sequences of microsatellites and the chitinase gene of L. infantum, L. donovani, and L. archibaldi strains provided strong support for monophyly of most strains of Sudanese origin, regardless of their species classification (Noyes HA, unpublished data). ${ }^{3}$ 
TABLE 2

List of primers used

\begin{tabular}{|c|c|c|}
\hline A-01 5'-CAGGCCCTTC-3' & B-01 5'-GTTTCGCTCC-3' & 192 5'-GCAAGTCACT-3' \\
\hline A-02 5'-TGCCGAGCTG-3' & B-02 5'-TGATCCCTGG-3' & 193 5'-TGCTGGCTTT-3' \\
\hline A-03 5'-AGTCAGCCAC-3' & B-03 5'-CATCCCCCTG-3' & 181 5'-ATGACGACGG-3' \\
\hline A-04 5'-AATCGGGCTG-3' & B-04 5'-GGACTGGAGT-3' & 160 5'-CGATTCAGAG-3' \\
\hline A-05 5'-AGGGGTCTTG-3' & B-05 5'-TGCGCCCTTC-3' & 198 5'-GCAGGACTGC-3' \\
\hline A-06 5'-GGTCCCTGAC-3' & B-06 5'-TGCTCTGCCC-3' & 159 5'-GAGCCCGTAG-3' \\
\hline A-07 5'-GAAACGGGTG-3' & B-07 5'-GGTGACGCAG-3' & 164 5'-CCAAGATGCT-3' \\
\hline A-08 5'-GTGACGTAGG-3' & B-08 5'-GTCCACACGG-3' & ILO875 5'-GTCCGTGAGC-3' \\
\hline A-09 5'-GGGTAACGCC-3' & B-09 5'-TGGGGGACTC-3' & ILO872 5'-CCCGCCATCT-3' \\
\hline A-10 5'-GTGATCGCAG-3' & B-10 5'-CTGCTGGGAC-3' & TAF300 5'-CACCTCAAAACATACCCC-3' \\
\hline A-11 5'-CAATCGCCGT-3' & B-11 5'-GTAGACCCGT-3' & TA610 5'-TCAACCGATTACAAACCA-3' \\
\hline A-12 5'-TCGGCGATAG-3' & B-12 5'-CCTTGACGCA-3' & TA150 5'-ATGCGATGAGTGGTTGAG-3' \\
\hline A-13 5'-CAGCACCCAC-3' & B-13 5'-TTCCCCCGCT-3' & PLID2-9 5'-CAAAAGTCCCCACCAATCCC-3' \\
\hline A-14 5'-TCTGTGCTGG-3' & B-14 5'-TCCGCTCTGG-3' & C2 5'-GAAACGGGTG-3' \\
\hline A-15 5'-TTCCGAACCC-3' & B-15 5'-GGAGGGTGTT-3' & C4 5'-AATCGGGCTG-3' \\
\hline A-16 5'-AGCCAGCGAA-3' & B-16 5'-TTTGCCCGGA-3' & C5 5'-CTCACGTAGG-3' \\
\hline A-17 5'-GACCGCTTGT-3' & B-17 5'-AGGGAACGAG-3' & C6 5'-CTGATCGCAG-3' \\
\hline A-18 5'-AGGTGACCGT-3' & B-18 5'-CCACAGCAGT-3' & D8 5'-AGCCAGCGAA-3' \\
\hline A-19 5'-CAAACGTCGG-3' & B-19 5'-ACCCCCGAAG-3' & D10 5'-GTTGCGATCC-3' \\
\hline A-20 5'-GTTGCGATCC-3' & B-20 5'-GGACCCTTAC-3' & H1 5'-CGCGCCCGCT-3' \\
\hline H4 5'-TGCCGAGCTG-3' & & L2 5'-CGGACGTCGC-3' \\
\hline
\end{tabular}

Leishmania infantum (thus defined as excluding MON-30) was not monophyletic due to the position of strains LEM 189 and BUCK. These two strains were genetically distant from the other $L$. infantum and the trees constrained for mono-

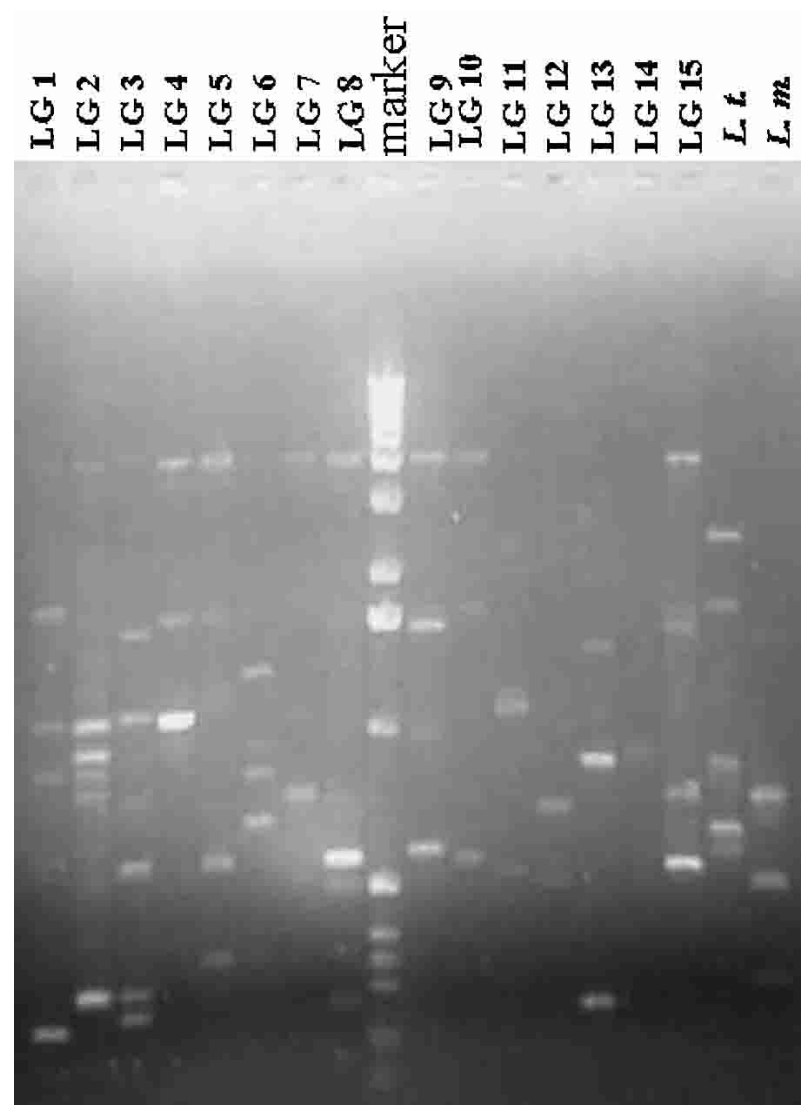

FiguRE 1. Random amplified polymorphic DNA amplification patterns of Leishmania spp. strains (Table 1) with primer B20. Marker = 100-basepair ladder (Gibco-BRL, Gaithersburg, MD); L. $t .=$ L. tropica; $=$ L. m. $=$ L. major. phyly of $L$. infantum had significantly lower bootstrap support than unconstrained trees. This is an unexpected result, since every method used so far to study the $L$. donovani complex has shown that L. infantum without MON-30 strains

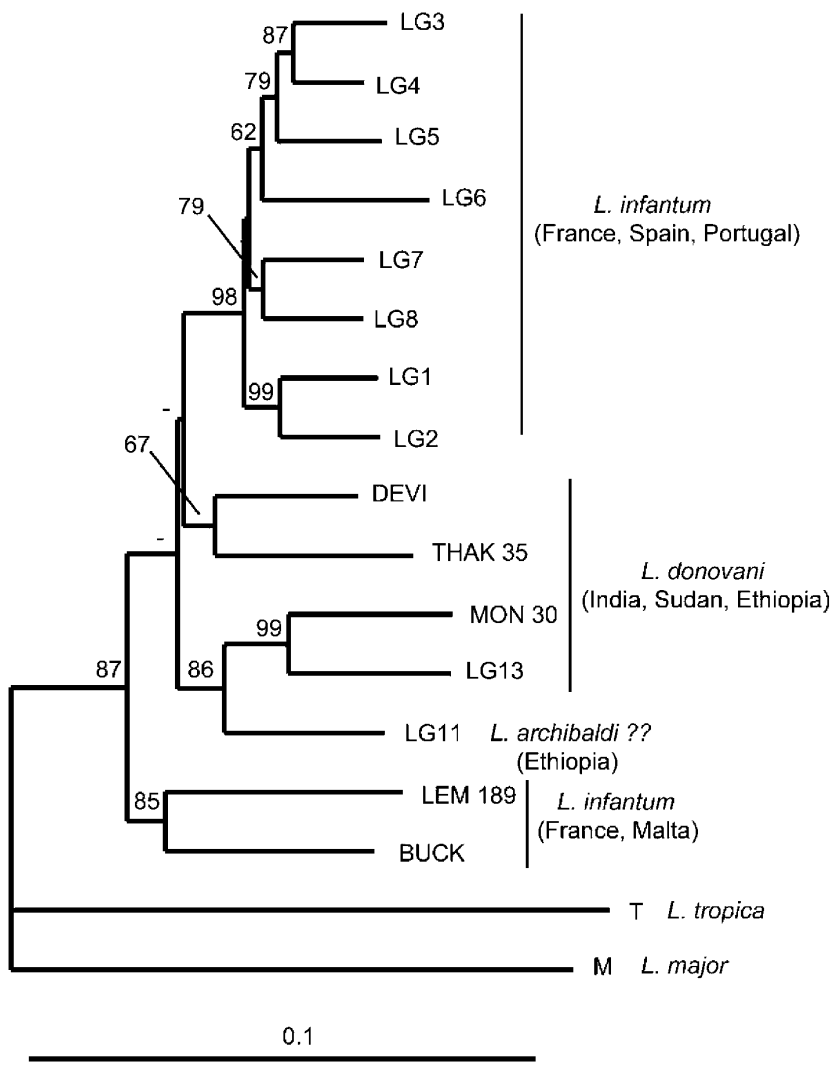

FIGURE 2. Phylogenetic tree constructed using minimum evolution as an optimality criterion and UpHolt distance matrices, with Leishmania major and L. tropica used as outgroups. The bootstrap test was carried out using 1,000 replicates. The scale for substitution/ site is given under the tree. The geographic origin of the strains is indicated. 

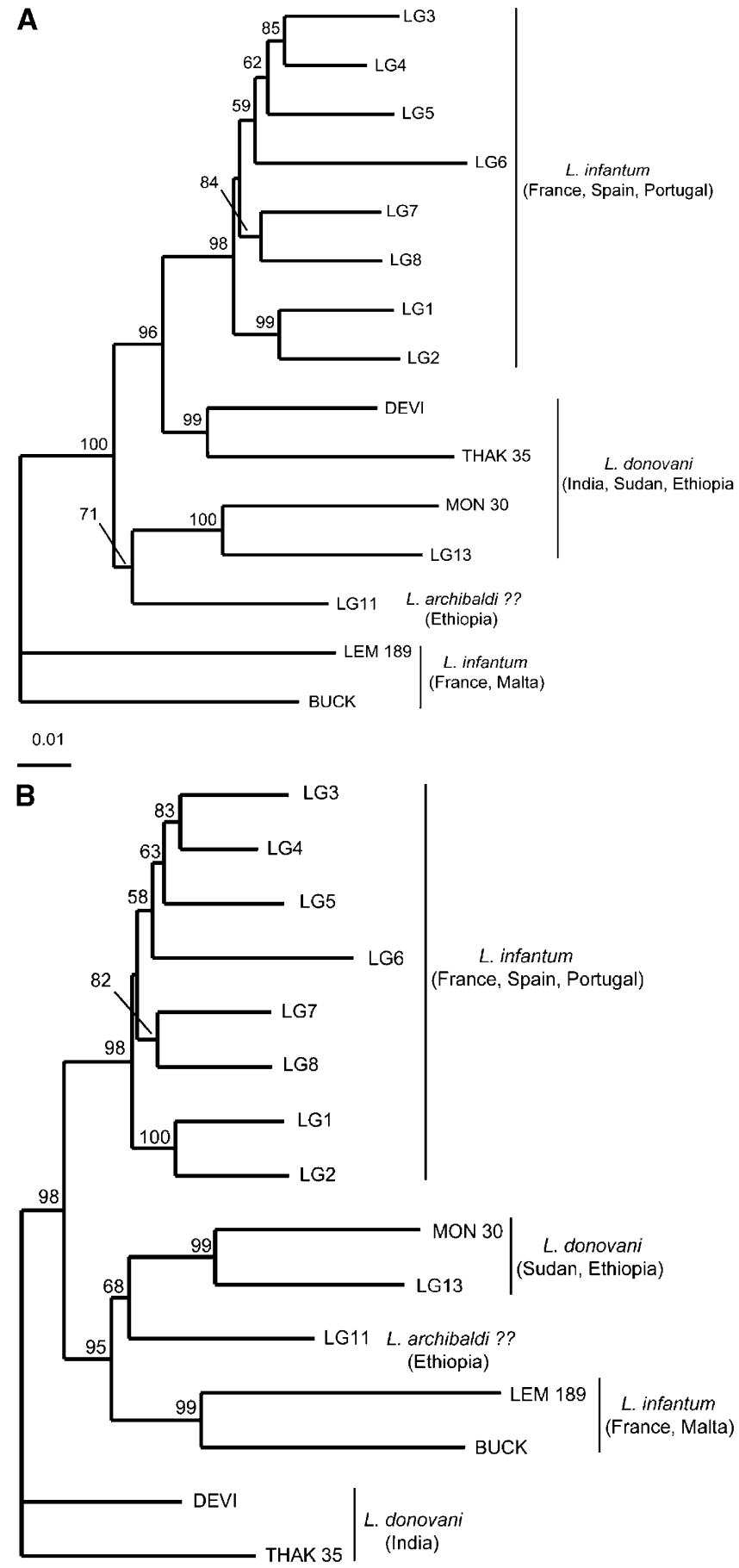

0.01

FIgURE 3. Phylogenetic trees constructed using minimum evolution as an optimality criterion and $\mathbf{A}$, UpHolt distance matrices or $\mathbf{B}$, NeiLi distance matrices, with the dataset narrowed to the Leishmania donovani group only. The bootstrap test was carried out using 1,000 replicates. The scale for substitution/site is given under the tree. The geographic origin of the strains is indicated. Leishmania infantum strains LEM 189 and BUCK served as outgroups in $\mathbf{A}$ and L. donovani strains DEVI and THAK 35 served as outgroups in $\mathbf{B}$.

is indeed monophyletic. ${ }^{6,9,15}$ It should be stressed, however, that strain BUCK seems to be one of the most divergent strains in this species. ${ }^{9}$

It should be emphasized that current taxonomy of the $L$. donovani complex relies on differences in the mobility of selected enzymes in starch gels and that this mobility can be altered by a single point mutation. As such, multilocus enzyme electrophoresis-based taxonomies rely on a small number of characteristics, in contrast with the relationships among parasites proposed here, which are based on hundreds of different characteristics. Unfortunately, only a relatively small number of strains have so far been extensively studied by molecular means and much remains to be done. However, we present here more data that support a call for a taxonomic re-evaluation of the L. donovani complex.

Received May 30, 2003. Accepted for publication January 22, 2004.

Acknowledgments: We thank Jean-Pierre Dedet and Francine Pratlong (Laboratoire d'Ecologie Médicale et Pathologie Parasitaire, Montpellier, France) and Jan Votýpka (Department of Parasitology, Charles University, Prague, Czech Republic) for providing the strains, and Ivan Fiala (Institute of Parasitology, České Budčjovice, Czech Republic) for help with phylogeny.

Financial support: This work was supported by grants from the European Community (QLK2-CT-2001-01810) and the Ministry of Education of the Czech Republic (MSMT-123100003).

Authors' addresses: Eva Zemanová, Milan Jirků, and Julius Lukeš, Institute of Parasitology, Czech Academy of Sciences, and Faculty of Biology, University of South Bohemia, Branišovská 31, 37005, Ceské Budějovice, Telephone: 420-38-777-5416, Fax: 420-38-531-0388, E-mail: jula@paru.cas.cz. Isabel L. Mauricio and Michael A. Miles, Pathogen Molecular Biology and Biochemistry Unit, Department of Infectious and Tropical Diseases, London School of Hygiene and Tropical Medicine, Keppel Street, London WC1E 7HT, United Kingdom.

Reprint requests: Julius Lukeš, Department of Molecular Parasitology, Institute of Parasitology, Czech Academy of Sciences, Branišovská 31, 370 05, Ceské Budějovice, Czech Republic.

\section{REFERENCES}

1. Rioux JA, Lanotte G, Serres E, Pratlong F, Bastien P, Perieres J, 1990. Taxonomy of Leishmania. Use of isoenzymes. Suggestions for a new classification. Ann Parasitol Hum Comp 65: 111-125.

2. Chouicha N, Lanotte G, Pratlong F, Cuba CAC, Velez ID, Dedet JP, 1997. Phylogenetic taxonomy of Leishmania (Viannia) braziliensis based on isoenzymatic study of 137 isolates. Parasitology 115: 343-348.

3. Lewin S, Schönian G, El Tai N, Oskam L, Bastien P, Presber W, 2002. Strain typing in Leishmania donovani by using sequenceconfirmed amplified region analysis. Int J Parasitol 32: 1267 1276.

4. El-Tai NO, El Fari M, Mauricio IL, Miles MA, Oskam L, El Safi SH, Presber W, Schönian G, 2001. Leishmania donovani: intraspecific polymorphisms of Sudanese isolates revealed by PCR-based analyses and DNA sequencing. Exp Parasitol 97: 35-44.

5. Fernandes O, Murthy VK, Kurath U, Degrave WM, Campbell DA, 1994. Mini-exon gene variation in human pathogenic Leishmania species. Mol Biochem Parasitol 66: 261-271.

6. Mauricio IL, Howard MK, Stothard JR, Miles MA, 1999. Genomic diversity in the Leishmania donovani complex. Parasitology 119: 237-246.

7. Jamjoom MB, Ashford RW, Bates PA, Kemp SJ, Noyes HA, 2002. Towards a standard battery of microsatellite markers for the analysis of the Leishmania donovani complex. Ann Trop Med Parasitol 96: 265-270.

8. Morales MA, Chicharro C, Ares M, Canavate C, Barker DC, Alvar J, 2001. Molecular tracking of infections by Leishmania infantum. Trans R Soc Trop Med Hyg 95: 104-107.

9. Mauricio IL, Gaunt MW, Stothard JR, Miles MA, 2001. Genetic typing and phylogeny of the Leishmania donovani complex by 
restriction analysis of PCR amplified gp63 intergenic regions. Parasitology 122: 393-403.

10. Guerbouj S, Victoir K, Guizani I, Seridi N, Nuwayri-Salti N, Belkaid M, Ben Ismail R, LeRay D, Dujardin J-C, 2001. Gp63 gene polymorphism and population structure of Leishmania donovani complex: influence of the host selection pressure? Parasitology 122: 25-35.

11. Banuls AL, Guerrini F, Le Pont F, Barrera C, Espinel I, Guderian R, Echeverria R, Tibayrenc M, 1997. Evidence for hybridization by multilocus enzyme electrophoresis and random amplified polymorphic DNA between Leishmania braziliensis and Leishmania panamensis/guyanensis in Ecuador. $J$ Eukaryot Microbiol 44: 408-411.

12. Schönian G, Akuffo H, Lewin S, Maasho K, Nylén S, Pratlong F, Eisenberger CL, Schnur LF, Presber W, 2000. Genetic variability within the species Leishmania aethiopica does not cor- relate with clinical variations of cutaneous leishmaniasis. $\mathrm{Mol}$ Biochem Parasitol 106: 239-248.

13. Toledo A, Martín-Sánchez J, Pesson B, Sanchiz-Marín C, Morillas-Márquez F, 2002. Genetic variability within the species Leishmania infantum by RAPD. (2002) A lack of correlation with zymodeme structure. Mol Biochem Parasitol 119: 257264.

14. Guizani I, Dellagi K, Ben Ismail R, 2002. Random amplified polymorphic DNA technique for identification and differentiation of old world Leishmania species. Am J Trop Med Hyg 66: $152-156$.

15. Hide M, Banuls AL, Tibayrenc M, 2001. Genetic heterogeneity and phylogenetic status of Leishmania (Leishmania) infantum zymodeme MON-1: epidemiological implications. Parasitology 123: 425-432. 